\title{
A Relaxation Two-Sweep Modulus-Based Matrix Splitting Iteration Method for Linear Complementarity Problems
}

\author{
Xiaofei Peng, Meng Wang and Wen $\mathrm{Li}^{*}$ \\ School of Mathematical Sciences, South China Normal University, Guangzhou, \\ 510631, China.
}

Received 02 March 2018; Accepted (in revised version) 22 June 2018.

\begin{abstract}
A general RTMS iteration method for linear complementarity problems is proposed. Choosing various pairs of relaxation parameters, we obtain new two-sweep modulus-based matrix splitting iteration methods and already known iteration procedures such as the MS [1] and TMS [27] iteration methods. If the system matrix is positive definite or an $H_{+}$-matrix and the relaxation parameters $\omega_{1}$ and $\omega_{2}$ satisfy the inequality $0 \leq \omega_{1}, \omega_{2} \leq 1$, sufficient conditions for the uniform convergence of MS, TMS and NTMS iteration methods are established. Numerical results show that with quasi-optimal parameters, RTMS iteration method outperforms MS and TMS iteration methods in terms of computing efficiency.
\end{abstract}

AMS subject classifications: 65F10, 65F35, 65H10

Key words: Linear complementarity problem, matrix splitting, iteration method, relaxation, convergence.

\section{Introduction}

Let $\mathbf{R}^{n}$ and $\mathbf{R}^{n \times n}$ be, respectively, the $n$-dimensional real vector and matrix spaces. For a matrix $A \in \mathbf{R}^{n \times n}$ and a vector $q \in \mathbf{R}^{n}$, the linear complementarity problem, abbreviated as $\operatorname{LCP}(q, A)$, consists in finding the pair of vectors $w, z \in \mathbb{R}^{n}$, such that

$$
w:=A z+q \geq 0, \quad z \geq 0 \quad \text { and } \quad z^{\top} w=0,
$$

where $\mathbf{T}$ denotes the transposition operation. The $\operatorname{LCP}(q, A)$ often arises in applications such as free boundary problems, network equilibrium, contact problems - cf. [7, 10, 22] and the references therein. To solve the $\operatorname{LCP}(q, A)$, van Bokhoven [24] reformulated it as an implicit fixed-point equation. The procedure, called the modulus method, was modified by Dong and Jiang [9] by including a shifting parameter into iteration process. Bai [1]

\footnotetext{
*Corresponding author. Email addresses: liwen@scnu.edu.cn (W. Li), pxf6628@163.com (X. Peng), vm_0802@163.com (M. Wang)
} 
established a modulus-based matrix splitting (MS) iteration method, based on a more effective and economical matrix splitting technique in actual computation. Zhang and Ren [29] proved the convergence of MS iteration method under a weak condition and Li [15] considered MS iteration method under more general conditions. The accelerated overrelaxation types of MS iteration methods have been studied in $[8,12]$ and the best diagonal matrix-parameter for such approaches has been determined. Further generalisations of the modulus-based matrix splitting iteration methods are connected with special matrix splittings $[14,20,30,32]$, preconditioning technique $[16,28]$ or relaxation strategies [26,31]. Moreover, the modulus-based synchronous multisplitting and modulus-based synchronous two-stage multisplitting iteration methods, aimed at the high parallel computational efficiency are developed and analysed $[2,3,17]$. On the other hand, modulusbased MS iteration methods have been applied to nonlinear complementarity problems cf. $[13,18,19,21]$.

Here, starting from the two-sweep modulus-based matrix splitting (TMS) iteration method in Ref. [27], we consider a relaxation two-sweep modulus-based matrix splitting iteration method and prove its convergence for $H_{+}$and positive definite system matrices, where relaxation strategy is different from [31]. This new method includes MS iteration method [1] and TMS iteration method [27] as its special cases and contains new two-sweep modulus-based matrix splitting iteration methods. Moreover, numerical results show its superiority over similar methods, both in number of iterations and CPU time.

The rest of this paper is organised as follows. In Section 2 we provide necessary definitions and auxiliary results. The relaxation two-sweep modulus-based matrix splitting iteration method is introduced in Section 3 and its convergence is studied in Section 4. Numerical results are discussed in Section 5. Section 6 contains concluding remarks.

\section{Preliminaries}

Most of material presented in this sections can be found in Refs. [6, 7, 11,22, 25].

If $A=\left(a_{i j}\right)$ and $B=\left(b_{i j}\right)$ are real $m \times n$ matrices, then the inequality $A \geq B(A>B)$ means that $a_{i j} \geq b_{i j}\left(a_{i j}>b_{i j}\right)$ for all $i$ and $j$. Subsequently, matrix $A=\left(a_{i j}\right)$ is called non-negative (positive) if $a_{i j} \geq 0\left(a_{i j}>0\right)$ for all $i$ and $j$. Besides, for any $A \in \mathbb{R}^{m \times n}$ by $|A|$ we denote the matrix $\left(\left|a_{i j}\right|\right)$.

Let $A$ be a square matrix and $\operatorname{sp}(A)$ refer to the spectrum, $\rho(A)$ to the spectral radius and $\operatorname{diag}(A)$ to the diagonal part of $A$. Moreover, the comparison matrix $\langle A\rangle=\left(\left\langle a_{i j}\right\rangle\right)$ for $A$ is the one with the entries $\left\langle a_{i j}\right\rangle=\left|a_{i j}\right|$ if $i=j$ and $\left\langle a_{i j}\right\rangle=-\left|a_{i j}\right|$ if $i \neq j$. The matrix $A$ is called $Z$-matrix if all off-diagonal entries of $A$ are non-positive, $M$-matrix if it is a $Z$-matrix with $A^{-1} \geq 0$ and $H$-matrix if its comparison matrix $\langle A\rangle$ is an $M$-matrix. Besides, any $H$-matrix with positive diagonal entries is called $H_{+}$-matrix.

The representation $A=M-N$ is called:

1. The splitting of the matrix $A$ if $M$ is a nonsingular matrix.

2. Convergent splitting if $\rho\left(M^{-1} N\right)<1$. 
3. $M$-splitting if $M$ is a nonsingular $M$-matrix and $N \geq 0$.

4. $H$-splitting if $\langle M\rangle-|N|$ is an $M$-matrix.

5. $H$-compatible splitting if $\langle A\rangle=\langle M\rangle-|N|$.

We note the $H$-compatible splitting of an $H$-matrix is also an $H$-splitting, but not vice versa. If $A=M-N$ is an $M$-splitting and $A$ is a nonsingular $M$-matrix, then $\rho\left(M^{-1} N\right)<1$. Further, a $Z$-matrix $A$ is an $M$-matrix if and only if there exists a positive vector $v$ such that $A v>0$. If $A$ is an $M$-matrix and $B$ is a $Z$-matrix, then $B \geq A$ implies that $B$ is an $M$-matrix. Let us recall that any $H$-matrix $A$ has the property $\left|A^{-1}\right| \leq\langle A\rangle^{-1}$. If $A$ is a non-negative matrix and there are a positive vector $v$ and a positive constant $\theta$ such that $A v \leq \theta v(A v<\theta v)$, then $\rho(A) \leq \theta(\rho(A)<\theta)$.

A matrix $A$ is called a $P$-matrix if all its principal minors are positive. It turns that a matrix $A$ is a $P$-matrix if and only if the corresponding $\operatorname{LCP}(q, A)$ has a unique solution for any $q \in \mathbf{R}^{n}$. We note that any $H_{+}$-matrix and any positive definite matrix are $P$-matrices cf. $[4,5,7]$.

Let us now recall auxiliary results needed in what follows.

Lemma 2.1 (cf. Shen \& Huang [23]). If

$$
W=\left[\begin{array}{ll}
F & G \\
I & 0
\end{array}\right] \geq 0 \text { and } \rho(F+G)<1
$$

then $\rho(W)<1$.

Lemma 2.2 (cf. Berman and \& Plemmons [6]). Let $A=D-L-U:=D-B$, where $D, L$ and $U$ are, respectively, diagonal, strictly lower-triangular and strictly upper-triangular matrices of the matrix $A$. If $a_{i i} \neq 0$ for all $1 \leq i \leq n$, then $A$ is an $H$-matrix if and only if $\rho\left(|D|^{-1}|B|\right)<1$, where $D^{-1} B$ is the Jacobi matrix associated with $A$.

\section{A Relaxation Two-Sweep Splitting Iteration Method}

Let $\Omega$ be a positive diagonal matrix and $\gamma$ a positive constant. Setting $z:=(1 / \gamma)(x+|x|)$, $w:=1 /(\gamma) \Omega(|x|-x)$ and $A=M-N$, we rewrite the LCP (1.1) as the system of fixed-point equations

$$
(\Omega+M) x=N x+(\Omega-A)|x|-\gamma q .
$$

Using this representation (3.1), Bai [1] and Wu et al. [27] proposed the following iteration methods for the $\operatorname{LCP}(q, A)$.

Method 3.1 (cf. [1], MS Iteration Method). Let $A=M-N$ be a splitting of $A$. Given an initial vector $x^{(0)} \in \mathbf{R}^{n}$, compute $x^{(k)} \in \mathbf{R}^{n}$ by solving the linear system

$$
(\Omega+M) x^{(k+1)}=N x^{(k)}+(\Omega-A)\left|x^{(k)}\right|-\gamma q,
$$


and set

$$
z^{(k+1)}=\frac{1}{\gamma}\left(\left|x^{(k+1)}\right|+x^{(k+1)}\right)
$$

for $k=1,2, \cdots$ until the iteration sequence $\left\{z^{(k)}\right\}_{k=1}^{+\infty} \subset \mathbf{R}^{n}$ converges.

Method 3.2 (cf. [27], TMS Iteration Method). Let $A=M-N$ be a splitting of $A$. Given two initial vectors $x^{(0)}, x^{(1)} \in \mathbf{R}^{n}$, compute $x^{(k)} \in \mathbf{R}^{n}$ by solving the linear system

$$
(\Omega+M) x^{(k+1)}=N x^{(k)}+(\Omega-A)\left|x^{(k-1)}\right|-\gamma q,
$$

and set

$$
z^{(k+1)}=\frac{1}{\gamma}\left(\left|x^{(k+1)}\right|+x^{(k+1)}\right)
$$

for $k=1,2, \cdots$ until the iteration sequence $\left\{z^{(k)}\right\}_{k=1}^{+\infty} \subset \mathbf{R}^{n}$ converges.

It is easily seen that the only difference between Methods 3.1 and 3.2 is the structure of the iterative processes - viz. the iterations in Methods 3.1 and 3.2 are, respectively, spanned on two and three successive steps.

Interchanging $x^{(k)}$ and $x^{(k-1)}$ in (3.2), we obtain a new two-sweep modulus-based matrix splitting (NTMS) iteration method.

Method 3.3 (NTMS Iteration Method). Let $A=M-N$ be a splitting of $A$. Given two initial vectors $x^{(0)}, x^{(1)} \in \mathbf{R}^{n}$, compute $x^{(k)} \in \mathbf{R}^{n}$ by solving the linear system

$$
(\Omega+M) x^{(k+1)}=N x^{(k-1)}+(\Omega-A)\left|x^{(k)}\right|-\gamma q,
$$

and set

$$
z^{(k+1)}=\frac{1}{\gamma}\left(\left|x^{(k+1)}\right|+x^{(k+1)}\right)
$$

for $k=1,2, \cdots$ until the iteration sequence $\left\{z^{(k)}\right\}_{k=1}^{+\infty} \subset \mathbf{R}^{n}$ converges.

Moreover, one can consider a relaxation two-sweep modulus-based matrix splitting (RTMS) iteration method containing two additional relaxation parameters $\omega_{1}$ and $\omega_{2}$.

Method 3.4 (RTMS Iteration Method). Let $A=M-N$ be a splitting of $A$. Given two nonnegative constants $\omega_{1}$ and $\omega_{2}$ and two initial vectors $x^{(0)}, x^{(1)} \in \mathbf{R}^{n}$, compute $x^{(k)} \in \mathbf{R}^{n}$ by solving the linear system

$$
(\Omega+M) x^{(k+1)}=N\left[\omega_{1} x^{(k)}+\left(1-\omega_{1}\right) x^{(k-1)}\right]+(\Omega-A)\left[\left(1-\omega_{2}\right)\left|x^{(k)}\right|+\omega_{2}\left|x^{(k-1)}\right|\right]-\gamma q,
$$

and set

$$
z^{(k+1)}=\frac{1}{\gamma}\left(\left|x^{(k+1)}\right|+x^{(k+1)}\right)
$$

for $k=1,2, \cdots$ until the iteration sequence $\left\{z^{(k)}\right\}_{k=1}^{+\infty} \subset \mathbf{R}^{n}$ converges. 
Let $\alpha \neq 0$ and $\beta$ be real numbers and let

$$
M:=\frac{1}{\alpha}(D-\beta L), \quad N:=\frac{1}{\alpha}((1-\alpha) D+(\alpha-\beta) L+\alpha U), \quad \gamma:=2 .
$$

With this special splitting, Method 3.4 produces a relaxation two-sweep modulus-based accelerated overrelaxation (RTMAOR) iteration method. If $\alpha=\beta \neq 0, \alpha=\beta=1$ and $\alpha=1, \beta=0$, the RTMAOR iteration method becomes, respectively, the relaxation twosweep modulus-based successive overrelaxation (RTMSOR) iteration method, the relaxation two-sweep modulus-based Guess-Seidel (RTMGS) iteration method and the relaxation two-sweep modulus-based Jacobi (RTMJ) iteration method. In particular, for $\alpha \neq 0$, $\beta=1$ and $\alpha \neq 0, \beta=0$, the RTMAOR iteration method becomes the relaxation two-sweep modulus-based Extrapolated Guess-Seidel (RTMEGS) iteration method and the relaxation two-sweep modulus-based Extrapolated Jacobi (RTMEJ) iteration method, respectively.

Remark 3.1. Method 3.4 provides a general framework for modulus-based matrix splitting iteration methods for the $\operatorname{LCP}(q, A)$. In particular,

- Method 3.1 is obtained from Method 3.4 by setting $\omega_{1}=1, \omega_{2}=0$ in (3.3). Correspondingly, RTMAOR, RTMSOR, RTMEGS and RTMEJ iteration methods transform into MAOR, MSOR, MEGS and MEJ iteration methods, respectively.

- Method 3.2 is obtained from Method 3.4 by setting $\omega_{1}=\omega_{2}=1$. Correspondingly, RTMAOR, RTMSOR, RTMEGS and RTMEJ iteration methods transform into TMAOR, TMSOR, TMEGS and TMEJ iteration methods, respectively.

- Method 3.3 is obtained from Method 3.4 by setting $\omega_{1}=\omega_{2}=0$. Correspondingly, RTMAOR, RTMSOR, RTMEGS and RTMEJ iteration methods transform into new TMAOR (NTMAOR), new TMSOR (NTMSOR), new TMEGS (NTMEGS) and new TMEJ (NTMEJ) iteration methods, respectively.

\section{Convergence Analysis}

In this section, we establish the convergence of Method 3.4 for positive definite and $H_{+}$-matrices $A$. In what follows, we will use the notation $m_{i}=\max \left\{2 \omega_{i}-1,1\right\}, i=1,2$. Moreover, we consider the matrices $F$ and $G$ defined by

$$
\begin{aligned}
& F:=\omega_{1}\left|(\Omega+M)^{-1} N\right|+\left|1-\omega_{2}\right|\left|(\Omega+M)^{-1}(\Omega-A)\right|, \\
& G:=\left|1-\omega_{1}\right|\left|(\Omega+M)^{-1} N\right|+\omega_{2}\left|(\Omega+M)^{-1}(\Omega-A)\right| .
\end{aligned}
$$

Let $\left(z_{*}, w_{*}\right)$ be a solution of the $\operatorname{LCP}(q, A)$. According to [1, Theorem 2.1] and (3.1), the vector $x_{*}:=(\gamma / 2)\left(z_{*}-\Omega^{-1} w_{*}\right)$ satisfies the implicit fixed-point equation

$$
(\Omega+M) x_{*}=N\left[\omega_{1} x_{*}+\left(1-\omega_{1}\right) x_{*}\right]+(\Omega-A)\left[\left(1-\omega_{2}\right)\left|x_{*}\right|+\omega_{2}\left|x_{*}\right|\right]-\gamma q,
$$


where $\left|x_{*}\right|:=(\gamma / 2)\left(z_{*}+\Omega^{-1} w_{*}\right)$. Subtracting (4.2) from (3.3) yields

$$
\begin{aligned}
x^{(k+1)}-x_{*}= & (\Omega+M)^{-1}\left[\omega_{1} N\left(x^{(k)}-x_{*}\right)+\left(1-\omega_{2}\right)(\Omega-A)\left(\left|x^{(k)}\right|-\left|x_{*}\right|\right)\right. \\
& \left.+\left(1-\omega_{1}\right) N\left(x^{(k-1)}-x_{*}\right)+\omega_{2}(\Omega-A)\left(\left|x^{(k-1)}\right|-\left|x_{*}\right|\right)\right],
\end{aligned}
$$

and using the triangle inequality ||$x^{(k)}|-| x_{*}|| \leq\left|x^{(k)}-x_{*}\right|$, we obtain

$$
\left|x^{(k+1)}-x_{*}\right| \leq F\left|x^{(k)}-x_{*}\right|+G\left|x^{(k-1)}-x_{*}\right|
$$

with the matrices $F$ and $G$ defined by (4.1). Adding the identical relation

$$
\left|x^{(k)}-x_{*}\right|=\left|x^{(k)}-x_{*}\right|
$$

to the inequality red (4.3), we can write the obtained system in the form

$$
\left|\left[\begin{array}{c}
x^{(k+1)}-x_{*} \\
x^{(k)}-x_{*}
\end{array}\right]\right| \leq\left[\begin{array}{cc}
F & G \\
I & 0
\end{array}\right]\left|\left[\begin{array}{c}
x^{(k)}-x_{*} \\
x^{(k-1)}-x_{*}
\end{array}\right]\right| .
$$

It is clear that the convergence of the iteration sequence $\left\{x^{(k)}\right\}_{k=1}^{+\infty} \subset \mathbf{R}^{n}$ generated by Method 3.4 implies convergence of the sequence $\left\{z^{(k)}\right\}_{k=1}^{+\infty}$. However, according to Lemma 2.1, one has

$$
W=\left[\begin{array}{ll}
F & G \\
I & 0
\end{array}\right] \geq 0
$$

and due to the inequality (4.4), we only need to show that $\rho(F+G)<1$.

\subsection{Positive definite matrices}

We first consider the convergence of Method 3.4 in the case of positive definite system matrices $A$.

Theorem 4.1. Let $M \in \mathbf{R}^{n \times n}$ be a positive definite matrix and $A=M-N$ be a splitting of the positive definite matrix $A \in \mathbf{R}^{n \times n}$. Consider a positive diagonal matrix $\Omega \in \mathbf{R}^{n \times n}$ and non-negative constants $\omega_{1}, \omega_{2}$ and the terms

$$
\begin{aligned}
& \xi(\Omega)=\left\|\left|(\Omega+M)^{-1} N\right|\right\|, \\
& \eta(\Omega)=\left\|\left|(\Omega+M)^{-1}(\Omega-M)\right|\right\|, \\
& \delta(\Omega)=m_{2} \eta(\Omega)+\left(m_{1}+m_{2}\right) \xi(\Omega),
\end{aligned}
$$

where $\|\cdot\|$ is an arbitrary matrix norm and $m_{i}:=\max \left\{2 \omega_{i}-1,1\right\}, i=1,2$. If $\delta(\Omega)<$ 1 , then for any initial vectors $x^{(0)}, x^{(1)} \in \mathbf{R}^{n}$, the iteration sequence $\left\{z^{(k)}\right\}_{k=0}^{+\infty}$ generated by Method 3.4, converges to the exact solution $z_{*}$ of the $\operatorname{LCP}(q, A)$.

Proof. Since

$$
\left|(\Omega+M)^{-1}(\Omega-A)\right| \leq\left|(\Omega+M)^{-1}(\Omega-M)\right|+\left|(\Omega+M)^{-1} N\right|
$$


we have

$$
\begin{aligned}
\rho(F+G) & =\rho\left(m_{1}\left|(\Omega+M)^{-1} N\right|+m_{2}\left|(\Omega+M)^{-1}(\Omega-A)\right|\right) \\
& \leq \rho\left(\left(m_{1}+m_{2}\right)\left|(\Omega+M)^{-1} N\right|+m_{2}\left|(\Omega+M)^{-1}(\Omega-M)\right|\right) \\
& \leq\left\|\left(m_{1}+m_{2}\right)\left|(\Omega+M)^{-1} N\right|+m_{2}\left|(\Omega+M)^{-1}(\Omega-M)\right|\right\| \\
& \leq\left\|\left(m_{1}+m_{2}\right)\left|(\Omega+M)^{-1} N\right|\right\|+\left\|m_{2}\left|(\Omega+M)^{-1}(\Omega-M)\right|\right\| \\
& =\left(m_{1}+m_{2}\right) \xi(\Omega)+m_{2} \eta(\Omega)=\delta(\Omega) .
\end{aligned}
$$

The inequality $\delta(\Omega)<1$ yields $\rho(F+G)<1$ and the convergence of the sequence $\left\{z^{(k)}\right\}_{k=0}^{+\infty}$ follows.

Remark 4.1. Special cases of Theorem 4.1 produce a number of known results. For example:

- If $\omega_{1}=1$ and $\omega_{2}=0$, one obtains Theorem 4.1 in [1].

- If $\omega_{1}=\omega_{2}=1$, one obtains Theorem 4.1 in [27].

- If $\omega_{1}=\omega_{2}=0$, one obtain the convergence of Method 3.3 above.

Theorem 4.1 can be further specified as follows.

Theorem 4.2. Let $M \in \mathbf{R}^{n \times n}$ be a symmetric positive definite matrix, $A=M-N$ a splitting of the positive definite matrix $A \in \mathbf{R}^{n \times n}, \gamma$ a positive constant, $\Omega:=\omega I \in \mathbf{R}^{n \times n}$ with $\omega>$ $0, \tau:=\left\|M^{-1} N\right\|_{2}, m_{i}:=\max \left\{2 \omega_{i}-1,1\right\}$ with $\omega_{i} \geq 0, i=1,2$, and $\lambda_{\min }$ and $\lambda_{\max }$ be, respectively, the smallest and the largest eigenvalues of $M$. If the parameters $\omega, \omega_{1}, \omega_{2}$ satisfy one of the conditions

(I) $\omega \leq \sqrt{\lambda_{\min } \lambda_{\max }}$ and

$$
\left(m_{2}+1\right) \omega-\left[\left(m_{1}+m_{2}\right) \tau+m_{2}-1\right] \lambda_{\max }>0,
$$

(II) $\omega \geq \sqrt{\lambda_{\min } \lambda_{\max }}$ and

$$
\begin{aligned}
\left(m_{2}-1\right) \omega^{2} & +\left\{\left[\left(m_{2}-1\right)+\left(m_{1}+m_{2}\right) \tau\right] \lambda_{\max }-\left(m_{2}+1\right) \lambda_{\min }\right\} \omega \\
& +\left[\left(m_{1}+m_{2}\right) \tau-\left(m_{2}+1\right)\right] \lambda_{\min } \lambda_{\max }<0
\end{aligned}
$$

holds, then for any initial vectors $x^{(0)}, x^{(1)} \in \mathbf{R}^{n}$, the iteration sequence $\left\{z^{(k)}\right\}_{k=0}^{+\infty}$ generated by Method 3.4 converges to the unique solution $z_{*}$ of the $\operatorname{LCP}(q, A)$.

Proof. According to Theorem 4.1, we only have to show that $\delta(\Omega)<1$. Similar to the proof of [1, Theorem 4.2], we obtain

$$
\xi(\Omega)=\left\|(\Omega+M)^{-1} N\right\|_{2} \leq \frac{\lambda_{\max } \tau}{\omega+\lambda_{\max }}
$$


and

$$
\eta(\Omega)=\left\|(\Omega+M)^{-1}(\Omega-M)\right\|_{2}= \begin{cases}\frac{\lambda_{\max }-\omega}{\lambda_{\max }+\omega}, & \text { for } \omega \leq \sqrt{\lambda_{\min } \lambda_{\max }} \\ \frac{\omega-\lambda_{\min }}{\omega-\lambda_{\min }}, & \text { for } \omega \geq \sqrt{\lambda_{\min } \lambda_{\max }}\end{cases}
$$

It follows that

$$
\begin{aligned}
\delta(\Omega) & =\left(m_{1}+m_{2}\right) \xi(\Omega)+m_{2} \eta(\Omega) \\
& \leq\left\{\begin{array}{l}
\frac{\left[\left(m_{1}+m_{2}\right) \tau+m_{2}\right] \lambda_{\max }-m_{2} \omega}{\omega+\lambda_{\max }}, \text { for } \omega \leq \sqrt{\lambda_{\min } \lambda_{\max }}, \\
\frac{\left(m_{1}+m_{2}\right) \lambda_{\max } \tau}{\omega+\lambda_{\max }}+\frac{m_{2}\left(\omega-\lambda_{\min }\right)}{\omega+\lambda_{\min }}, \text { for } \omega \geq \sqrt{\lambda_{\min } \lambda_{\max }}
\end{array}\right.
\end{aligned}
$$

This inequality combined with either (4.5) or (4.6) yields estimate $\delta(\Omega)<1$.

Theorem 4.3. Assume that $0 \leq \omega_{2} \leq 1$ and all hypotheses of Theorem 4.2, except the relations (I) and (II), are satisfied. If one of the conditions

(III) $\tau<1, \tau^{2} \lambda_{\max }<\lambda_{\min }$ and

$$
0 \leq \omega_{1}<\frac{\sqrt{\lambda_{\min } \lambda_{\max }}}{\tau \lambda_{\max }}, \quad \max \left\{\omega_{1}, 1\right\} \tau \lambda_{\max }<\omega \leq \sqrt{\lambda_{\max } \lambda_{\min }}
$$

(IV) $\tau^{2} \lambda_{\max }<\lambda_{\min }<\tau \lambda_{\max }$ and

$$
0 \leq \omega_{1}<\frac{\sqrt{\lambda_{\min } \lambda_{\max }}}{\tau \lambda_{\max }}, \quad \sqrt{\lambda_{\max } \lambda_{\min }} \leq \omega<\frac{\left[1-\max \left\{\omega_{1}, 1\right\} \tau\right] \lambda_{\min } \lambda_{\max }}{\tau \lambda_{\max } \max \left\{\omega_{1}, 1\right\}-\lambda_{\min }}
$$

(V) $\tau<1, \tau \lambda_{\max } \leq \lambda_{\min }$ and

$$
0 \leq \omega_{1}<\frac{1}{\tau}, \quad \omega \geq \sqrt{\lambda_{\min } \lambda_{\max }}
$$

holds, then for any initial vectors $x^{(0)}, x^{(1)} \in \mathbf{R}^{n}$, the iteration sequence $\left\{z^{(k)}\right\}_{k=0}^{+\infty}$ generated by Method 3.4 converges to the unique solution $z_{*}$ of the $\operatorname{LCP}(q, A)$.

Proof. If $0 \leq \omega_{2} \leq 1$, then $m_{2}=\max \left\{2 \omega_{2}-1,1\right\}=1$. Moreover, since $m_{1}+1=$ $2 \max \left\{\omega_{1}, 1\right\}$, the inequalities (4.5) and (4.6) takes the form

$$
\begin{aligned}
& \omega>\tau \lambda_{\max } \max \left\{\omega_{1}, 1\right\}, \\
& {\left[\tau \lambda_{\max } \max \left\{\omega_{1}, 1\right\}-\lambda_{\min }\right] \omega-\left[1-\max \left\{\omega_{1}, 1\right\} \tau\right] \lambda_{\min } \lambda_{\max }<0 .}
\end{aligned}
$$

If $\omega$ and $\omega_{1}$ satisfy (4.10), then Condition (I) in Theorem 4.2 is equivalent to the second inequality in (4.7).

To solve the inequality (4.11), we consider two cases. 
Case (A) If $\max \left\{\omega_{1}, 1\right\} \tau \lambda_{\max }>\lambda_{\min }$ and $\omega$ and $\omega_{1}$ satisfy (4.11), then Condition (I) in Theorem 4.2 is equivalent to the second inequality in (4.8).

Case (B) If $\max \left\{\omega_{1}, 1\right\} \tau \lambda_{\max } \leq \lambda_{\min }$, then $1-\max \left\{\omega_{1}, 1\right\} \tau>0$ and the inequality (4.11) holds. Correspondingly, Condition (II) in Theorem 4.2 is equivalent to the second inequality in (4.9).

Since the obtained upper bound is not smaller than the corresponding lower bound, we immediately obtain conditions $(I I I)-(V)$.

Corollary 4.1. Assume that $0 \leq \omega_{i} \leq 1, i=1,2$ and all hypotheses of Theorem 4.2, except the relations (I) and (II), are satisfied. If one of the conditions

(VI) $\tau<1, \tau^{2} \lambda_{\max }<\lambda_{\min }$ and $\tau \lambda_{\max }<\omega \leq \sqrt{\lambda_{\max } \lambda_{\min }}$,

(VII) $\tau<1, \tau^{2} \lambda_{\max }<\lambda_{\min }<\tau \lambda_{\max }$ and $\sqrt{\lambda_{\max } \lambda_{\min }} \leq \omega<\frac{(1-\tau) \lambda_{\min } \lambda_{\max }}{\tau \lambda_{\max }-\lambda_{\min }}$,

(VIII) $\tau<1, \tau \lambda_{\max } \leq \lambda_{\min }$ and $\omega \geq \sqrt{\lambda_{\min } \lambda_{\max }}$

holds, then for any initial vectors $x^{(0)}, x^{(1)} \in \mathbf{R}^{n}$, the iteration sequence $\left\{z^{(k)}\right\}_{k=0}^{+\infty}$ generated by Method 3.4 converges to the unique solution $z_{*}$ of the $\operatorname{LCP}(q, A)$.

Remark 4.2. For Methods 3.1-3.3, the corresponding parameters $\omega_{1}, \omega_{2}$ satisfy the inequality $0 \leq \omega_{1}, \omega_{2} \leq 1-$ cf. Remark 3.1. Therefore, Corollary 4.1 provides the conditions of uniform convergence for all three methods in the case where system matrices are symmetric and positive definite. If $\omega_{1}=1, \omega_{2}=0$, Corollary 4.1 coincides with [1, Theorem 4.2]. For Methods 3.2-3.3 the convergence conditions are new.

\subsection{The case of $H_{+}$-matrix}

Now we consider the convergence of Method 3.4 for $H_{+}$system matrices.

Theorem 4.4. Let $A=D-B \in \mathbf{R}^{n \times n}$ be an $H_{+}$-matrix, $D=\operatorname{diag}(\mathrm{A}), A=M-N$ a splitting of $A$ and $m_{i}:=\max \left\{2 \omega_{i}-1,1\right\}, i=1,2$. If $\gamma$ is a positive constant, $\Omega \in \mathbf{R}^{n \times n}$ a positive diagonal matrix such that $\Omega \geq D$ and $\langle M\rangle-m_{1}|N|-\left(m_{2}-1\right) \Omega$ an $M$-matrix, then for any initial vectors $x^{(0)}, x^{(1)} \in \mathbf{R}^{n}$, the iteration sequence $\left\{z^{(k)}\right\}_{k=0}^{+\infty}$ generated by Method 3.4 converges to the unique solution $z_{*}$ of the $\operatorname{LCP}(q, A)$.

Proof. It is clear that $m_{i} \geq 1, i=1,2$ and since $\langle M\rangle-m_{1}|N|-\left(m_{2}-1\right) \Omega$ is an $M$-matrix and $A=M-N$ is the splitting of the $H_{+}$-matrix, one has

$$
\begin{aligned}
& a_{i i}=m_{i i}-n_{i i}>0, \\
& \left|m_{i i}\right|-m_{1}\left|n_{i i}\right|-\left(m_{2}-1\right) \omega_{i i}>0
\end{aligned}
$$

for all $i=1,2, \cdots, n$. The inequalities (4.12), (4.13) yield $m_{i i}>0$, and since $\langle M\rangle$ is an $M$-matrix, $\Omega+M$ is an $H_{+}$-matrix, as well. It follows that

$$
0 \leq\left|(\Omega+M)^{-1}\right| \leq(\Omega+\langle M\rangle)^{-1} .
$$


Recalling the condition $\Omega \geq D$, we write

$$
\begin{aligned}
F+G & =m_{1}\left|(\Omega+M)^{-1} N\right|+m_{2}\left|(\Omega+M)^{-1}(\Omega-A)\right| \\
& \leq\left|(\Omega+M)^{-1}\right|\left(m_{1}|N|+m_{2}|(\Omega-D)+B|\right) \\
& \leq(\Omega+\langle M\rangle)^{-1}\left(m_{1}|N|+m_{2} \Omega-m_{2}(D-|B|)\right. \\
& \leq I-(\Omega+\langle M\rangle)^{-1}\left(\langle M\rangle-m_{1}|N|-\left(m_{2}-1\right) \Omega+m_{2}(D-|B|) \triangleq \widetilde{W} .\right.
\end{aligned}
$$

Let us show the inequality $\rho(\widetilde{W})<1$. Since $\langle M\rangle-m_{1}|N|-\left(m_{2}-1\right) \Omega$ is an $M$-matrix, there is a positive vector $v$ such that

$$
\left(\langle M\rangle-m_{1}|N|-\left(m_{2}-1\right) \Omega\right) v>0
$$

In other words,

$$
\left(\left|m_{i i}\right|-m_{1}\left|n_{i i}\right|-\left(m_{2}-1\right) \omega_{i i}\right) v_{i}-\sum_{j \neq i}\left(\left|m_{i j}\right|+m_{1}\left|n_{i j}\right|\right) v_{j}>0
$$

for all $i=1,2, \cdots, n$. We observe that

$$
a_{i i}=\left|a_{i i}\right| \geq\left|m_{i i}\right|-\left|n_{i i}\right| \geq\left|m_{i i}\right|-m_{1}\left|n_{i i}\right|-\left(m_{2}-1\right) \omega_{i i}
$$

and

$$
\left|a_{i j}\right| \leq\left|m_{i j}\right|+\left|n_{i j}\right| \leq\left|m_{i j}\right|+m_{1}\left|n_{i j}\right| .
$$

It follows from (4.15) that

$$
m_{2}\left(a_{i i} v_{i}-\sum_{j \neq i}\left|a_{i j}\right| v_{j}\right)>0, \quad i=1,2, \cdots, n .
$$

Therefore,

$$
u \triangleq\left(\langle M\rangle-m_{1}|N|-\left(m_{2}-1\right) \Omega+m_{2}(D-|B|)\right) v>0 .
$$

Correspondingly,

$$
\widetilde{W} v=v-(\Omega+\langle M\rangle)^{-1} u<v,
$$

which implies the inequality $\rho(\widetilde{W})<1$, and the proof is completed.

$$
\begin{aligned}
& \text { If } 0 \leq \omega_{1}, \omega_{2} \leq 1 \text {, then } m_{1}=m_{2}=1 \text { and } \\
& \qquad\langle M\rangle-m_{1}|N|-\left(m_{2}-1\right) \Omega=\langle M\rangle-|N|,
\end{aligned}
$$

and we can rewrite Theorem 4.4 as follows.

Corollary 4.2. Let $A \in \mathbf{R}^{n \times n}$ be an $H_{+}$-matrix, $D=\operatorname{diag}(\mathrm{A}), A=M-N$ an $H$-splitting of $A$ and $0 \leq \omega_{i} \leq 1, i=1$, 2. If $\gamma$ is a positive constant and $\Omega \in \mathbf{R}^{n \times n}$ a positive diagonal matrix such that $\Omega \geq D$, then for any initial vectors $x^{(0)}, x^{(1)} \in \mathbf{R}^{n}$, the iteration sequence $\left\{z^{(k)}\right\}_{k=0}^{+\infty}$ generated by Method 3.4 converges to the unique solution $z_{*}$ of the $\operatorname{LCP}(q, A)$. 
Remark 4.3. For Methods 3.1-3.3, the corresponding parameters $\omega_{1}, \omega_{2}$ satisfy the inequality $0 \leq \omega_{1}, \omega_{2} \leq 1-$ cf. Remark 3.1. Therefore, Corollary 4.2 provides the conditions of uniform convergence for these methods. Moreover, with the corresponding choice of $\omega_{1}$ and $\omega_{2}$, Corollary 4.2 coincides with [29, Theorem 3.1] or with [27, Theorem 4.2].

We can also establish the convergence of the RTMAOR iteration method.

Theorem 4.5. Assume that the $H_{+}$-matrix $A \in \mathbf{R}^{n \times n}$ is represented in the form $A=D-L-U:=$ $D-B$, where $D$ is diagonal, $L$ strictly lower-triangular and $U$ strictly upper-triangular matrices of the matrix $A$. Choose a positive constant $\gamma$ and a positive diagonal matrix $\Omega \in \mathbf{R}^{n \times n}$ such that $\Omega \geq D$ and set $m_{1}=\max \left\{2 \omega_{1}-1,1\right\}$. If the conditions

$$
\begin{aligned}
& 0 \leq \omega_{1}<\frac{1}{\rho\left(D^{-1}|B|\right)}, \quad 0 \leq \omega_{2} \leq 1, \quad \text { and } \quad 0 \leq \beta \leq \alpha \\
& \frac{m_{1}-1}{\left(m_{1}+1\right)\left(1-\rho\left(D^{-1}|B|\right)\right)}<\alpha<\frac{m_{1}+1}{m_{1}-1+\left(m_{1}+1\right) \rho\left(D^{-1}|B|\right)}
\end{aligned}
$$

hold, then the RTMAOR iteration method converges for any initial vectors $x^{(0)}, x^{(1)} \in \mathbf{R}^{n}$.

Proof. From the proof of Theorem 4.4, the method converges if $\rho(\widetilde{W})<1$, where $\widetilde{W}$ is defined by (4.14). We note that the $M$ and $N$ in the RTMAOR iteration method are presented in (3.4). Since $m_{2}=\max \left\{2 \omega_{2}-1,1\right\}=1$ and $m_{1}=\max \left\{2 \omega_{1}-1,1\right\} \geq 1$, we have

$$
\begin{aligned}
& \langle M\rangle-m_{1}|N|-\left(m_{2}-1\right) \Omega+m_{2}(D-|B|)=\langle M\rangle-m_{1}|N|+D-|B| \\
= & \frac{1}{\alpha}\left(D-\beta|L|-m_{1}(|1-\alpha| D+(\alpha-\beta)|L|+\alpha|U|)+\alpha(D-|B|)\right) \\
= & \frac{1}{\alpha}\left[\left(1+\alpha-|1-\alpha| m_{1}\right) D-\left(\alpha+\beta+(\alpha-\beta) m_{1}\right)|L|-\left(m_{1}+1\right) \alpha|U|\right] \\
\geq & \frac{1}{\alpha}\left[\left(1+\alpha-|1-\alpha| m_{1}\right) D-\left(m_{1}+1\right) \alpha|B|\right] \triangleq \frac{1}{\alpha} \widehat{A} .
\end{aligned}
$$

Recalling that $\Omega \geq D$ and $A$ is an $H_{+}$-matrix, we observe that $\alpha \Omega+D-\beta|L|$ is an $M$-matrix too. Therefore,

$$
(\alpha \Omega+D-\beta|L|)^{-1}>(\alpha \Omega+D)^{-1}
$$

and the inequalities (4.18) and (4.19) yield

$$
\begin{aligned}
\widetilde{W} & =I-(\Omega+\langle M\rangle)^{-1}\left(\langle M\rangle-m_{1}|N|-\left(m_{2}-1\right) \Omega+m_{2}(D-|B|)\right. \\
& \leq I-(\alpha \Omega+D-\beta|L|)^{-1} \widehat{A}<I-(\alpha \Omega+D)^{-1} \widehat{A} \triangleq \widehat{W} .
\end{aligned}
$$

It remains to show that $\rho(\widehat{W})<1$. By Lemma $2.2, \widehat{A}$ is an $H$-matrix if and only if

$$
\rho\left(D^{-1}|B|\right)<\frac{|1+\alpha-| 1-\alpha\left|m_{1}\right|}{\alpha\left(m_{1}+1\right)} .
$$


The inequality (4.18) implies that $\widehat{A}$ is a $Z$-matrix. Therefore, $\widehat{A}$ is an $M$-matrix if and only if $\widehat{A}$ has positive diagonal entries and the inequality (4.21) holds - i.e. if

$$
0<\rho\left(D^{-1}|B|\right)<\frac{1+\alpha-|1-\alpha| m_{1}}{\alpha\left(m_{1}+1\right)} .
$$

Straightforward calculations show that (4.22) is satisfied if and only if

or

$$
\frac{m_{1}-1}{\left(m_{1}+1\right)\left(1-\rho\left(D^{-1}|B|\right)\right)}<\alpha \leq 1
$$

$$
1<\alpha<\frac{m_{1}+1}{m_{1}-1+\left(m_{1}+1\right) \rho\left(D^{-1}|B|\right)} .
$$

However, (4.23) and (4.24) compose the condition (4.17). Moreover, since $\omega_{1}$ satisfies conditions (4.16) and $A$ is an $H_{+}$-matrix, we have

$$
\rho\left(D^{-1}|B|\right)<1 \text { and } \rho\left(D^{-1}|B|\right) \omega_{1}<1 .
$$

It follows that $\rho\left(D^{-1}|B|\right)\left(m_{1}+1\right)<2$. Therefore, the corresponding upper bound is at least the corresponding lower bound in (4.17). So far we proved that $\widehat{A}$ is an $M$-matrix. Let us now show that $\rho(\widehat{W})<1$. Since $\widehat{A}$ is an $M$-matrix, there is a positive vector $v>0$ such that $u \triangleq \widehat{A} v>0$. Consequently,

$$
\widehat{W} v=v-(\alpha \Omega+D)^{-1} u<v,
$$

and the inequalities $\widehat{W} v \geq 0,(\alpha \Omega+D)^{-1} u>0$ yield $\rho(\widehat{W})<1$. It follows from (4.20) that $\rho(\widetilde{W})<1$.

Remark 4.4. If $\omega_{1}$ and $\omega_{2}$ satisfy the conditions (4.16), then under the conditions of Theorem 4.5, RTMJ and RTMGS iteration methods converge. On the other hand, RTMSOR, RTMEJ and RTMEGS iteration methods converge if $\omega_{1}, \omega_{2}$ and $\alpha$ satisfy the corresponding inequalities (4.16) and (4.17).

Further, if $0 \leq \omega_{1}, \omega_{2} \leq 1$, Theorem 4.5 gives the following convergence results.

Corollary 4.3. Let $0 \leq \omega_{i} \leq 1, i=1,2$ and the $H_{+}$-matrix $A \in \mathbf{R}^{n \times n}$ be represented in the form $A=D-L-U:=D-B$, where $D$ is diagonal, $L$ strictly lower-triangular and $U$ strictly upper-triangular matrices of the matrix $A$. Choose a positive constant $\gamma$ and a positive diagonal matrix $\Omega \in \mathbf{R}^{n \times n}$ such that $\Omega \geq D$. If the condition

$$
0<\alpha<\frac{1}{\rho\left(D^{-1}|B|\right)} \text { and } 0 \leq \beta \leq \alpha
$$

holds, then for any initial vectors $x^{(0)}, x^{(1)} \in \mathbf{R}^{n}$, the RTMAOR iteration method converges.

Remark 4.5. Assume that $0 \leq \beta \leq \alpha$.

- If $\omega_{1}=1$ and $\omega_{2}=0$, then Corollary 4.3 provides the convergence domain for the parameter $\alpha$ in the MAOR iterations, coinciding with the first item in [8, Relation (3.2)].

- If $\omega_{1}=\omega_{2}=1$, Corollary 4.3 coincides with [27, Theorem 4.4].

- If $\omega_{1}=\omega_{2}=0$, Corollary 4.3 provides the conditions of convergence for NTMAOR iteration method. 


\section{Numerical Examples}

In this section, we consider two examples that show the effectiveness of the relaxation two-sweep modulus-based matrix splitting iteration methods. Note that the number of iteration steps, the elapsed CPU time (in seconds) and the norm of absolute residual vectors are, respectively, denoted by 'IT', 'CPU' and 'RES'. All initial vectors in our tests are the same - viz.

$$
x^{(0)}=x^{(1)}=(1,0,1,0, \cdots, 1,0, \cdots)^{\boldsymbol{\top}} \in \mathbf{R}^{n} .
$$

The computations are performed in MATLAB environment with double machine precision. The iterations are terminated if

$$
\mathrm{RES}=\left\|\min \left(A z^{(i)}+q, z^{(i)}\right)\right\|_{2} \leq 10^{-5}
$$

where $z^{(i)}$ is the $i$ th approximate solution to the $\operatorname{LCP}(q, A)$ and the minimum is taken componentwise. The notation "- " is used in the case, if the stop criteria is not satisfied within 1500 iterations.

We will test the methods in Remark 3.1. They can be distributed into four groups — viz. MAOR, TMAOR, NTMAOR and RTMAOR methods, according to the choice of the parameters $\omega_{i}, i=1,2$. Actually, the methods from the first three groups are special cases of the methods from the fourth group. On the other hand, these methods can also be distributed in three groups: MSOR type, the MEGS type and the MEJ type methods, according to the choice of parameter $\beta-$ cf. Table 1 .

Table 1: Classification of testing methods.

\begin{tabular}{||c|c|c|c|c||}
\hline method & MAOR & TMAOR & NTMAOR & RTMAOR \\
\hline MSOR type & MSOR & TMSOR & NTMSOR & RTMSOR \\
\hline MEGS type & MEGS & TMEGS & NTMEGS & RTMEGS \\
\hline MEJ type & MEGS & TMEJ & NTMEJ & RTMEJ \\
\hline
\end{tabular}

Example 5.1 (cf. Dong \& Jiang [9]). Let $m$ be a positive integer, $n=m^{2}$ and $\mu$ a nonnegative constant. Consider the $\operatorname{LCP}(q, A)$ with the matrix $A=\hat{A}+\mu I$ and vector $q=-A z_{*} \in \mathbf{R}^{n}$, where

$$
\hat{A}=\left(\begin{array}{cccccc}
S & -I & & & & \\
-I & S & -I & & & \\
& -I & S & \ddots & & \\
& & \ddots & \ddots & -I & \\
& & & -I & S & -I \\
& & & & -I & S
\end{array}\right) \in \mathbf{R}^{n \times n}
$$

is a block-tridiagonal matrix, $S=\operatorname{tridiag}(-1,4,-1) \in \mathbf{R}^{m \times m}$ a tridiagonal matrix, and $z_{*}=$ $(1,2,1,2, \cdots, 1,2, \cdots)^{T} \in \mathbb{R}^{n}$ the unique solution of the $\operatorname{LCP}(q, A)$. 
Obviously, the system matrix $A$ in Example 5.1 is symmetric and positive definite and thus the corresponding $\operatorname{LCP}(q, A)$ has a unique solution.

Example 5.2 (cf. Bai [1]). Let $m$ be a positive integer, $n=m^{2}$ and $\mu$ a nonnegative constant. Consider the $\operatorname{LCP}(q, A)$ with the matrix $A=\hat{A}+\mu I$ and vector $q=-A z_{*} \in \mathbf{R}^{n}$, where

$$
\hat{A}=\left(\begin{array}{cccccc}
S & -0.5 I & & & & \\
-1.5 I & S & -0.5 I & & & \\
& -1.5 I & S & \ddots & & \\
& & \ddots & \ddots & -0.5 I & \\
& & & -1.5 I & S & -0.5 I \\
& & & & -1.5 I & S
\end{array}\right) \in \mathbf{R}^{n \times n}
$$

is a block-tridiagonal matrix, $S=\operatorname{tridiag}(-1.5,4,-0.5) \in \mathbf{R}^{m \times m}$ a tridiagonal matrix, and $z_{*}=(1,2,1,2, \cdots, 1,2, \cdots)^{T} \in \mathbb{R}^{n}$ the unique solution of the $\operatorname{LCP}(q, A)$.

It is easily seen that the system matrix $A$ in Example 5.2 is an $H_{+}$-matrix, and thus the corresponding $\operatorname{LCP}(q, A)$ has a unique solution.

The numerical results with different problem sizes $n=m^{2}$ and $\Omega=D, \omega_{2}=0$ and $\omega_{1} \in[0,1]$ are reported in Tables 3-5. The parameters $\alpha_{* 1}, \alpha_{* 2}$ and $\alpha_{* 3}$ are, respectively, the quasi-optimal values of $\alpha$ in special cases of the iteration methods MAOR, TMAOR and NTMAOR. The quasi-optimal values of $\alpha$ and $\omega_{1}$ in the special cases of the RTMAOR iteration method are denoted by $\alpha_{*}$ and $\omega_{1 *}$, respectively. These quasi-optimal parameters are chosen to minimise the iteration steps for the problem of the smallest size $m=40$. Then they are used in associated larger problems, where $m=80$ and $m=120-$ cf. Table 2 .

From Tables 2-5 we have the following observations and remarks.

(1) In all numerical tests for Examples 5.1-5.2, the special RTMAOR methods are always superior to the methods MAOR, TMAOR and NTMAOR in terms of iteration steps and elapsed CPU time. In addition, for the weakly diagonally dominant matrix $A$, the special NTMAOR methods are more effective than MAOR and TMAOR methods. For the strongly diagonally dominant matrix $A$, the special MAOR iteration methods outperform TMAOR and NTMAOR iteration methods.

(2) Excluding the case $\mu=0$, Tables 3-5 suggest that all these methods have convergence rate nearly independent of the scale of the test problems, which is a very useful property of iterative methods. It allows to experimentally obtain the quasi-optimal parameters by testing small size problems.

(3) For nonsymmetric system matrices, Tables 3-4 show that, NTMSOR and NTMEGS methods have almost the same convergence rate as MSOR and MEGS iteration methods. Besides, Table 5 demonstrates that for larger $\mu$, TMEJ method is more competitive than NTMEJ one. In particular, it follows from Tables 2 and 5 that MJ method - i.e. MEJ method with $\alpha=1$ is the best. 
Table 2: Quasi-optimal parameters for Examples 5.1-5.2, $m=40$.

\begin{tabular}{||c|c|c|c|c||}
\hline Method & $\mu$ & & Example 5.1 & Example 5.2 \\
\hline MSOR type & 0 & $\left(\alpha_{* 1}, \alpha_{* 2}, \alpha_{* 3}\right)$ & $(2.2,2.2,3.5)$ & $(2.5,1.6,2.1)$ \\
& & $\left(\alpha_{*}, \omega_{* 1}\right)$ & $(4.3,0.6)$ & $(3.4,0.6)$ \\
\cline { 2 - 5 } & 2 & $\left(\alpha_{* 1}, \alpha_{* 2}, \alpha_{* 3}\right)$ & $(1.3,1.6,1.4)$ & $(1.4,1.2,1.3)$ \\
& & $\left(\alpha_{*}, \omega_{* 1}\right)$ & $(2.0,0.7)$ & $(1.5,0.6)$ \\
\cline { 2 - 5 } & 4 & $\left(\alpha_{* 1}, \alpha_{* 2}, \alpha_{* 3}\right)$ & $(1.2,1.4,1.2)$ & $(1.2,1.2,1.2)$ \\
& & $\left(\alpha_{*}, \omega_{* 1}\right)$ & $(1.5,0.7)$ & $(1.3,0.7)$ \\
\hline MEGS type & 0.5 & $\left(\alpha_{* 1}, \alpha_{* 2}, \alpha_{* 3}\right)$ & $(1.3,3.8,2.1)$ & $(1.5,3.0,2.1)$ \\
& & $\left(\alpha_{*}, \omega_{* 1}\right)$ & $(3.9,0.5)$ & $(3.6,0.5)$ \\
\cline { 2 - 5 } & 1.5 & $\left(\alpha_{* 1}, \alpha_{* 2}, \alpha_{* 3}\right)$ & $(1.2,2.6,1.5)$ & $(1.3,1.9,1.5)$ \\
& & $\left(\alpha_{*}, \omega_{* 1}\right)$ & $(2.7,0.6)$ & $(2.2,0.6)$ \\
\cline { 2 - 5 } & 2.5 & $\left(\alpha_{* 1}, \alpha_{* 2}, \alpha_{* 3}\right)$ & $(1.2,1.9,1.4)$ & $(1.2,1.5,1.3)$ \\
& & $\left(\alpha_{*}, \omega_{* 1}\right)$ & $(2.1,0.7)$ & $(1.7,0.6)$ \\
\hline MEJ type & 0.5 & $\left(\alpha_{* 1}, \alpha_{* 2}, \alpha_{* 3}\right)$ & $(1.0,4.3,1.7)$ & $(1.0,3.8,1.6)$ \\
& & $\left(\alpha_{*}, \omega_{* 1}\right)$ & $(4.3,0.5)$ & $(2.7,0.6)$ \\
\cline { 2 - 5 } & 1.5 & $\left(\alpha_{* 1}, \alpha_{* 2}, \alpha_{* 3}\right)$ & $(1.0,3.9,1.5)$ & $(1.0,3.6,1.4)$ \\
& & $\left(\alpha_{*}, \omega_{* 1}\right)$ & $(2.7,0.6)$ & $(2.2,0.6)$ \\
\cline { 2 - 5 } & 2.5 & $\left(\alpha_{* 1}, \alpha_{* 2}, \alpha_{* 3}\right)$ & $(1.0,2.8,1.3)$ & $(1.0,2.8,1.3)$ \\
& & $\left(\alpha_{*}, \omega_{* 1}\right)$ & $(2.1,0.7)$ & $(1.9,0.7)$ \\
\hline
\end{tabular}

\section{Concluding Remarks}

We used a relaxation strategy to introduce an RTMS iteration method. Choosing various pairs of relaxation parameters, we obtain new two-sweep modulus-based matrix splitting iteration methods and already known iteration procedures such as the MS [1] and TMS [27] iteration methods. If the system matrix is positive definite or an $H_{+}$-matrix and the relaxation parameters $\omega_{1}$ and $\omega_{2}$ satisfy the inequality $0 \leq \omega_{1}, \omega_{2} \leq 1$, sufficient conditions for the uniform convergence of MS, TMS and NTMS iteration methods are established. Numerical results show that with quasi-optimal parameters, RTMS iteration method outperforms MS and TMS iteration methods in terms of computing efficiency. Note that the finding of optimal relaxation parameters is a challenging problem. Here, they are determined by numerical tests for small size problems.

\section{Acknowledgments}

The authors would like to thank the anonymous referees for their constructive comments and suggestions, which greatly improved the paper. Sincere thanks to the editor Prof. Victor D. Didenko for his careful reading and helpful suggestions.

The work was supported by the National Natural Science Foundation of China (Grants Nos. 11571124, 11671158, 11801097), the Mayor Project No 2016KZDXM025 and by the Innovation Team Project No. 2015KCXTD007 of Guangdong Provincial General University. 
Table 3: Numerical results for MSOR type methods.

\begin{tabular}{|c|c|c|c|c|c|c|c|c|}
\hline & \multirow[b]{2}{*}{$m$} & \multirow[b]{2}{*}{ Method } & \multicolumn{3}{|c|}{ Example 5.1} & \multicolumn{3}{|c|}{ Example 5.2} \\
\hline & & & IT & $\mathrm{CPU}$ & RES & IT & CPU & RES \\
\hline \multirow{12}{*}{$\mu=0$} & \multirow[t]{4}{*}{40} & MSOR & 1408 & 0.5386 & $9.9 \mathrm{e}-06$ & 94 & 0.0375 & $9.9 \mathrm{e}-06$ \\
\hline & & TMSOR & - & - & - & 266 & 0.1026 & $9.5 e-06$ \\
\hline & & NTMSOR & 639 & 0.2484 & $9.9 e-06$ & 57 & 0.0224 & 7.9e-06 \\
\hline & & RTMSOR & 412 & 0.1814 & $9.9 \mathrm{e}-06$ & 43 & 0.0191 & $9.4 \mathrm{e}-06$ \\
\hline & \multirow[t]{4}{*}{80} & MSOR & - & - & - & 162 & 0.2621 & $8.2 \mathrm{e}-06$ \\
\hline & & TMSOR & - & - & - & 514 & 0.8240 & $9.1 \mathrm{e}-06$ \\
\hline & & NTMSOR & - & - & - & 93 & 0.1488 & $9.0 \mathrm{e}-06$ \\
\hline & & RTMSOR & - & - & - & 78 & 0.1380 & $6.5 e-06$ \\
\hline & \multirow[t]{4}{*}{120} & MSOR & - & - & - & 225 & 0.8319 & $9.1 \mathrm{e}-06$ \\
\hline & & TMSOR & - & - & - & 785 & 2.8603 & 8.7e-06 \\
\hline & & NTMSOR & - & - & - & 138 & 0.5311 & $9.2 \mathrm{e}-06$ \\
\hline & & RTMSOR & - & - & - & 111 & 0.4423 & $6.5 \mathrm{e}-06$ \\
\hline \multirow[t]{12}{*}{$\mu=2$} & \multirow[t]{4}{*}{40} & MSOR & 26 & 0.0101 & $7.2 \mathrm{e}-06$ & 20 & 0.0083 & $7.9 \mathrm{e}-06$ \\
\hline & & TMSOR & 43 & 0.0173 & 8.7e-06 & 45 & 0.0182 & 7.0e-06 \\
\hline & & NTMSOR & 26 & 0.0113 & $8.3 e-06$ & 18 & 0.0070 & $7.2 \mathrm{e}-06$ \\
\hline & & RTMSOR & 16 & 0.0082 & $8.8 \mathrm{e}-06$ & 17 & 0.0077 & 7.3e-06 \\
\hline & \multirow[t]{4}{*}{80} & MSOR & 27 & 0.0431 & $7.9 \mathrm{e}-06$ & 21 & 0.0368 & $7.9 \mathrm{e}-06$ \\
\hline & & TMSOR & 45 & 0.0717 & $8.4 \mathrm{e}-06$ & 47 & 0.0801 & $8.2 \mathrm{e}-06$ \\
\hline & & NTMSOR & 28 & 0.0459 & $5.7 e-06$ & 19 & 0.0323 & $5.5 e-06$ \\
\hline & & RTMSOR & 17 & 0.0290 & $5.2 \mathrm{e}-06$ & 18 & 0.0322 & 7.0e-06 \\
\hline & \multirow[t]{4}{*}{120} & MSOR & 28 & 0.1068 & $6.4 \mathrm{e}-06$ & 22 & 0.0841 & $5.5 \mathrm{e}-06$ \\
\hline & & TMSOR & 47 & 0.1779 & 7.0e-06 & 49 & 0.1951 & $6.5 \mathrm{e}-06$ \\
\hline & & NTMSOR & 28 & 0.1112 & $9.1 \mathrm{e}-06$ & 19 & 0.0721 & 8.1e-06 \\
\hline & & RTMSOR & 17 & 0.0696 & $7.2 \mathrm{e}-06$ & 19 & 0.0791 & $4.3 e-06$ \\
\hline \multirow[t]{12}{*}{$\mu=4$} & \multirow[t]{4}{*}{40} & MSOR & 17 & 0.0072 & $8.1 \mathrm{e}-06$ & 15 & 0.0062 & $3.8 \mathrm{e}-06$ \\
\hline & & TMSOR & 27 & 0.0115 & $9.6 \mathrm{e}-06$ & 27 & 0.0106 & $4.5 \mathrm{e}-06$ \\
\hline & & NTMSOR & 20 & 0.0086 & 7.1e-06 & 15 & 0.0061 & $5.8 \mathrm{e}-06$ \\
\hline & & RTMSOR & 13 & 0.0069 & $7.2 \mathrm{e}-06$ & 11 & 0.0048 & $5.4 \mathrm{e}-06$ \\
\hline & \multirow[t]{4}{*}{80} & MSOR & 18 & 0.0275 & $5.8 \mathrm{e}-06$ & 15 & 0.0224 & $8.4 \mathrm{e}-06$ \\
\hline & & TMSOR & 29 & 0.0446 & $4.8 \mathrm{e}-06$ & 27 & 0.0404 & 7.0e-06 \\
\hline & & NTMSOR & 21 & 0.0319 & 7.0e-06 & 15 & 0.0237 & $8.5 e-06$ \\
\hline & & RTMSOR & 14 & 0.0232 & $2.7 \mathrm{e}-06$ & 11 & 0.0190 & $8.6 e-06$ \\
\hline & \multirow[t]{4}{*}{120} & MSOR & 18 & 0.0673 & $8.4 \mathrm{e}-06$ & 16 & 0.0573 & $4.1 \mathrm{e}-06$ \\
\hline & & TMSOR & 29 & 0.1120 & $6.1 \mathrm{e}-06$ & 27 & 0.0943 & $9.2 \mathrm{e}-06$ \\
\hline & & NTMSOR & 22 & 0.0865 & $4.7 \mathrm{e}-06$ & 16 & 0.0569 & $6.0 \mathrm{e}-06$ \\
\hline & & RTMSOR & 14 & 0.0543 & $3.4 \mathrm{e}-06$ & 12 & 0.0471 & $4.4 \mathrm{e}-06$ \\
\hline
\end{tabular}


Table 4: Numerical results for MEGS type methods.

\begin{tabular}{|c|c|c|c|c|c|c|c|c|}
\hline & \multirow[b]{2}{*}{$m$} & \multirow[b]{2}{*}{ Method } & \multicolumn{3}{|c|}{ Example 5.1} & \multicolumn{3}{|c|}{ Example 5.2} \\
\hline & & & IT & CPU & RES & IT & CPU & RES \\
\hline \multirow[t]{12}{*}{$\mu=0.5$} & \multirow[t]{4}{*}{40} & MEGS & 83 & 0.0412 & $9.6 \mathrm{e}-06$ & 66 & 0.0264 & $8.4 \mathrm{e}-06$ \\
\hline & & TMEGS & 125 & 0.0513 & $9.8 \mathrm{e}-06$ & 113 & 0.0453 & $9.4 \mathrm{e}-06$ \\
\hline & & NTMEGS & 84 & 0.0344 & 8.0e-06 & 64 & 0.0252 & $9.3 e-06$ \\
\hline & & RTMEGS & 63 & 0.0275 & $9.8 \mathrm{e}-06$ & 54 & 0.0239 & $9.9 \mathrm{e}-06$ \\
\hline & \multirow[t]{4}{*}{80} & MEGS & 89 & 0.1426 & $9.1 \mathrm{e}-06$ & 73 & 0.1160 & $9.9 \mathrm{e}-06$ \\
\hline & & TMEGS & 134 & 0.2173 & $9.7 e-06$ & 127 & 0.2064 & $8.9 e-06$ \\
\hline & & NTMEGS & 89 & 0.1443 & $9.3 e-06$ & 72 & 0.1165 & $9.2 \mathrm{e}-06$ \\
\hline & & RTMEGS & 68 & 0.1182 & $9.0 \mathrm{e}-06$ & 61 & 0.1078 & $9.6 \mathrm{e}-06$ \\
\hline & \multirow[t]{4}{*}{120} & MEGS & 92 & 0.3370 & $8.9 e-06$ & 76 & 0.2771 & $9.9 \mathrm{e}-06$ \\
\hline & & TMEGS & 139 & 0.5220 & $9.0 \mathrm{e}-06$ & 133 & 0.5030 & $8.4 \mathrm{e}-06$ \\
\hline & & NTMEGS & 92 & 0.3476 & $9.2 \mathrm{e}-06$ & 75 & 0.2931 & $9.3 e-06$ \\
\hline & & RTMEGS & 70 & 0.2816 & $9.6 \mathrm{e}-06$ & 64 & 0.2569 & 8.9e-06 \\
\hline \multirow[t]{12}{*}{$\mu=1.5$} & \multirow[t]{4}{*}{40} & MEGS & 35 & 0.0140 & 9.0e-06 & 30 & 0.0117 & 7.0e-06 \\
\hline & & TMEGS & 51 & 0.0197 & $8.0 \mathrm{e}-06$ & 49 & 0.0189 & $8.4 \mathrm{e}-06$ \\
\hline & & NTMEGS & 38 & 0.0152 & $9.9 \mathrm{e}-06$ & 30 & 0.0119 & 7.1e-06 \\
\hline & & RTMEGS & 26 & 0.0114 & $9.1 \mathrm{e}-06$ & 24 & 0.0115 & $8.6 e-06$ \\
\hline & \multirow[t]{4}{*}{80} & MEGS & 37 & 0.0577 & $8.7 e-06$ & 32 & 0.0503 & $6.2 \mathrm{e}-06$ \\
\hline & & TMEGS & 53 & 0.0860 & $9.0 \mathrm{e}-06$ & 51 & 0.0813 & $8.5 e-06$ \\
\hline & & NTMEGS & 41 & 0.0669 & 7.0e-06 & 32 & 0.0523 & $6.4 \mathrm{e}-06$ \\
\hline & & RTMEGS & 28 & 0.0486 & $5.8 \mathrm{e}-06$ & 26 & 0.0443 & $6.1 \mathrm{e}-06$ \\
\hline & \multirow[t]{4}{*}{120} & MEGS & 38 & 0.1404 & $8.8 \mathrm{e}-06$ & 33 & 0.1215 & $6.0 \mathrm{e}-06$ \\
\hline & & TMEGS & 55 & 0.2042 & $6.9 \mathrm{e}-06$ & 53 & 0.1974 & $6.2 \mathrm{e}-06$ \\
\hline & & NTMEGS & 42 & 0.1565 & $7.4 \mathrm{e}-06$ & 33 & 0.1269 & $6.2 \mathrm{e}-06$ \\
\hline & & RTMEGS & 28 & 0.1124 & $8.8 \mathrm{e}-06$ & 27 & 0.1057 & $5.3 e-06$ \\
\hline \multirow[t]{12}{*}{$\mu=2.5$} & \multirow[t]{4}{*}{40} & MEGS & 24 & 0.0098 & $8.8 \mathrm{e}-06$ & 21 & 0.0082 & $9.3 e-06$ \\
\hline & & TMEGS & 37 & 0.0145 & $9.5 e-06$ & 37 & 0.0149 & $6.1 \mathrm{e}-06$ \\
\hline & & NTMEGS & 27 & 0.0111 & $9.1 \mathrm{e}-06$ & 22 & 0.0088 & $6.6 \mathrm{e}-06$ \\
\hline & & RTMEGS & 18 & 0.0084 & $8.7 e-06$ & 17 & 0.0074 & $9.6 \mathrm{e}-06$ \\
\hline & \multirow[t]{4}{*}{80} & MEGS & 25 & 0.0390 & $9.9 \mathrm{e}-06$ & 23 & 0.0373 & $4.7 e-06$ \\
\hline & & TMEGS & 39 & 0.0629 & $9.1 \mathrm{e}-06$ & 39 & 0.0627 & $6.0 \mathrm{e}-06$ \\
\hline & & NTMEGS & 28 & 0.0457 & 7.8e-06 & 23 & 0.0367 & 7.3e-06 \\
\hline & & RTMEGS & 19 & 0.0337 & $6.9 \mathrm{e}-06$ & 18 & 0.0320 & 7.1e-06 \\
\hline & \multirow[t]{4}{*}{120} & MEGS & 26 & 0.0971 & $7.9 \mathrm{e}-06$ & 23 & 0.0848 & $7.5 e-06$ \\
\hline & & TMEGS & 41 & 0.1491 & $6.0 \mathrm{e}-06$ & 39 & 0.1454 & $9.7 e-06$ \\
\hline & & NTMEGS & 29 & 0.1094 & 8.0e-06 & 24 & 0.0945 & 5.5e-06 \\
\hline & & RTMEGS & 20 & 0.0803 & $4.2 \mathrm{e}-06$ & 19 & 0.0806 & $4.1 \mathrm{e}-06$ \\
\hline
\end{tabular}


Table 5: Numerical results for MEJ type methods.

\begin{tabular}{|c|c|c|c|c|c|c|c|c|}
\hline & \multirow[b]{2}{*}{$m$} & \multirow[b]{2}{*}{ Method } & \multicolumn{3}{|c|}{ Example 5.1} & \multicolumn{3}{|c|}{ Example 5.2} \\
\hline & & & IT & CPU & RES & IT & CPU & RES \\
\hline \multirow[t]{12}{*}{$\mu=0.5$} & \multirow[t]{4}{*}{40} & MEJ & 119 & 1.8251 & $9.9 e-06$ & 111 & 0.0284 & $9.7 \mathrm{e}-06$ \\
\hline & & TMEJ & 130 & 0.0347 & $9.8 \mathrm{e}-06$ & 123 & 0.0330 & $9.8 \mathrm{e}-06$ \\
\hline & & NTMEJ & 127 & 0.0359 & $8.9 e-06$ & 122 & 0.0332 & $9.2 \mathrm{e}-06$ \\
\hline & & RTMEJ & 72 & 0.0223 & $9.9 e-06$ & 79 & 0.0253 & $9.7 \mathrm{e}-06$ \\
\hline & \multirow[t]{4}{*}{80} & MEJ & 128 & 0.1213 & $9.0 \mathrm{e}-06$ & 125 & 0.1174 & $9.2 \mathrm{e}-06$ \\
\hline & & TMEJ & 139 & 0.1387 & $9.9 \mathrm{e}-06$ & 139 & 0.1353 & $9.0 \mathrm{e}-06$ \\
\hline & & NTMEJ & 135 & 0.1336 & $9.7 \mathrm{e}-06$ & 137 & 0.1322 & $9.4 \mathrm{e}-06$ \\
\hline & & RTMEJ & 78 & 0.0914 & $8.4 \mathrm{e}-06$ & 89 & 0.0961 & $9.8 \mathrm{e}-06$ \\
\hline & \multirow[t]{4}{*}{120} & MEJ & 132 & 0.2775 & $9.1 \mathrm{e}-06$ & 130 & 0.2805 & $9.5 \mathrm{e}-06$ \\
\hline & & TMEJ & 144 & 0.3215 & $9.5 e-06$ & 144 & 0.3221 & $9.9 \mathrm{e}-06$ \\
\hline & & NTMEJ & 139 & 0.3120 & $9.8 \mathrm{e}-06$ & 143 & 0.3177 & $9.2 \mathrm{e}-06$ \\
\hline & & RTMEJ & 80 & 0.1988 & $9.5 e-06$ & 93 & 0.2325 & $9.6 \mathrm{e}-06$ \\
\hline \multirow[t]{12}{*}{$\mu=1.5$} & \multirow[t]{4}{*}{40} & MEJ & 49 & 0.0125 & $7.5 e-06$ & 48 & 0.0129 & $8.6 \mathrm{e}-06$ \\
\hline & & TMEJ & 51 & 0.0134 & $6.9 e-06$ & 53 & 0.0143 & $8.9 \mathrm{e}-06$ \\
\hline & & NTMEJ & 55 & 0.0144 & $6.9 \mathrm{e}-06$ & 56 & 0.0153 & 9.0e-06 \\
\hline & & RTMEJ & 32 & 0.0097 & $9.0 \mathrm{e}-06$ & 36 & 0.0113 & $7.8 \mathrm{e}-06$ \\
\hline & \multirow[t]{4}{*}{80} & MEJ & 51 & 0.0472 & $9.3 e-06$ & 51 & 0.0481 & $8.5 \mathrm{e}-06$ \\
\hline & & TMEJ & 53 & 0.0519 & 7.6e-06 & 57 & 0.0543 & 7.4e-06 \\
\hline & & NTMEJ & 57 & 0.0567 & $9.8 \mathrm{e}-06$ & 59 & 0.0569 & $9.9 \mathrm{e}-06$ \\
\hline & & RTMEJ & 34 & 0.0372 & 8.0e-06 & 38 & 0.0414 & 8.0e-06 \\
\hline & \multirow[t]{4}{*}{120} & MEJ & 53 & 0.1166 & 7.7e-06 & 53 & 0.1109 & $7.3 e-06$ \\
\hline & & TMEJ & 55 & 0.1242 & $6.2 \mathrm{e}-06$ & 59 & 0.1283 & $6.9 \mathrm{e}-06$ \\
\hline & & NTMEJ & 59 & 0.1316 & $9.8 \mathrm{e}-06$ & 61 & 0.1342 & $9.3 e-06$ \\
\hline & & RTMEJ & 35 & 0.0883 & $7.8 e-06$ & 39 & 0.0942 & $8.4 \mathrm{e}-06$ \\
\hline \multirow[t]{12}{*}{$\mu=2.5$} & \multirow[t]{4}{*}{40} & MEJ & 33 & 0.0090 & $8.8 \mathrm{e}-06$ & 33 & 0.0089 & $8.0 \mathrm{e}-06$ \\
\hline & & TMEJ & 37 & 0.0100 & $6.0 \mathrm{e}-06$ & 37 & 0.0098 & $8.4 \mathrm{e}-06$ \\
\hline & & NTMEJ & 40 & 0.0110 & 8.6e-06 & 40 & 0.0101 & $7.9 \mathrm{e}-06$ \\
\hline & & RTMEJ & 23 & 0.0072 & 8.0e-06 & 25 & 0.0073 & 7.3e-06 \\
\hline & \multirow[t]{4}{*}{80} & MEJ & 35 & 0.0335 & $7.5 e-06$ & 35 & 0.0341 & 7.1e-06 \\
\hline & & TMEJ & 39 & 0.0391 & $5.4 \mathrm{e}-06$ & 39 & 0.0376 & $6.71 \mathrm{e}-06$ \\
\hline & & NTMEJ & 42 & 0.0406 & 8.7e-06 & 42 & 0.0422 & $8.4 \mathrm{e}-06$ \\
\hline & & RTMEJ & 24 & 0.0293 & $9.1 \mathrm{e}-06$ & 26 & 0.0286 & $7.9 \mathrm{e}-06$ \\
\hline & \multirow[t]{4}{*}{120} & MEJ & 36 & 0.0747 & $7.2 \mathrm{e}-06$ & 36 & 0.0760 & $6.9 \mathrm{e}-06$ \\
\hline & & TMEJ & 39 & 0.0845 & $8.5 e-06$ & 39 & 0.0851 & $9.8 \mathrm{e}-06$ \\
\hline & & NTMEJ & 43 & 0.0919 & $9.1 \mathrm{e}-06$ & 43 & 0.0942 & 9.0e-06 \\
\hline & & RTMEJ & 25 & 0.0635 & 7.1e-06 & 27 & 0.0660 & $6.3 e-06$ \\
\hline
\end{tabular}




\section{References}

[1] Z.-Z. Bai, Modulus-based matrix splitting iteration methods for linear complementarity problems, Numer. Linear Algebra Appl. 17, 917-933 (2010).

[2] Z.-Z. Bai and L.-L. Zhang, Modulus-based synchronous multisplitting iteration methods for linear complementarity problems, Numer. Linear Algebra Appl. 20, 425-439 (2013).

[3] Z.-Z. Bai and L.-L. Zhang, Modulus-based synchronous two-stage multisplitting iteration methods for linear complementarity problems, Numer. Algorithms 62, 59-77 (2013).

[4] Z.-Z. Bai, On the convergence of the multisplitting methods for the linear complementarity problem, SIAM J. Matrix Anal. Appl. 21, 67-78 (1999).

[5] Z.-Z. Bai and D. Evans, Matrix multisplitting methods with applications to linear complementarity problems: parallel asynchronous methods, Int. J. Comput. Math. 79, 205-232 (2002).

[6] A. Berman and R.J. Plemmons, Nonnegative Matrix in the Mathematical Sciences, Academic Press (1979).

[7] R.W. Cottle, J.-S. Pang and R.E. Stone, The Linear Complementarity Problem, Academic Press (1992).

[8] Lj. Cvetković, A. Hadjidimos and V. Kostić, On the choice of parameters in MAOR type splitting methods for the linear complementarity problem, Numer. Algorithms 67, 793-806 (2014).

[9] J.-L. Dong and M.-Q. Jiang, A modified modulus method for symmetric positive-definite linear complementarity problems, Numer. Linear Algebra Appl. 16, 129-143 (2009).

[10] M.C. Ferris and J.-S. Pang, Engineering and economic applications of complementarity problems, SIAM Rev. 39, 669-713 (1997).

[11] A. Frommer and G. Mayer, Convergence of relaxed parallel multisplitting methods, Linear Algebra Appl. 119, 141-152 (1989).

[12] A. Hadjidimos and M. Tzoumas, The solution of the linear complementarity problem by the matrix analogue of the accelerated overrelaxation iterative method, Numer. Algorithms 73, 665684 (2016).

[13] B.-H. Huang and C.-F. Ma, Accelerated modulus-based matrix splitting iteration method for a class of nonlinear complementarity problems, Computational and Applied Mathematics 37, 3053-3076 (2018).

[14] Y.F. Ke and C.F. Ma, On the convergence analysis of two-step modulus-based matrix splitting iteration method for linear complementarity problems, Appl. Math. Comput. 243, 413-418 (2014).

[15] W. Li, A general modulus-based matrix splitting method for linear complementarity problems of H-matrices, Appl. Math. Lett. 26, 1159-1164 (2013).

[16] W. Li and H. Zheng, A preconditioned modulus-based iteration method for solving linear complementarity problems of H-matrices, Linear Multilinear Algebra 64, 1390-1403 (2016).

[17] C.-L. Li and J.-T. Hong, Modulus-based synchronous multisplitting iteration methods for an implicit complementarity problem, East Asian J. Appl. Math. 7, 363-375 (2017).

[18] R. Li and J.-F. Yin, Accelerated modulus-based matrix splitting iteration methods for a restricted class of nonlinear complementarity problems, Numer. Algorithms 75, 339-358 (2017).

[19] R. Li, Y. Wang and J.-F. Yin, On the convergence of two-step modulus-based matrix splitting iteration methods for a restricted class of nonlinear complementarity problems with $\mathrm{H}_{+}$-matrices, Numer. Math. Theory Methods Appl. 11, 128-139 (2018).

[20] S.-M. Liu, H. Zheng and W. Li, A general accelerated modulus-based matrix splitting iteration method for solving linear complementarity problems, Calcolo 53, 189-199 (2016).

[21] C.-F. Ma and N. Huang, Modified modulus-based matrix splitting algorithms for a class of weakly nondifferentiable nonlinear complementarity problems, Appl. Numer. Math. 108, 116124 (2016). 
[22] K.G. Murty, Linear Complementarity, Linear and Nonlinear Programming, Heldermann Verlag (1988).

[23] S.-Q. Shen and T.-Z. Huang, Convergence and comparison theorems for double splittings of matrices, Computers and Mathematics with Applications 51, 1751-1760 (2006).

[24] W.M.G. van Bokhoven, Piecewise-linear Modelling and Analysis, Proefschrift (1981).

[25] R.S. Varga, Matrix Iterative Analysis, Prentice-Hall (1962).

[26] B.-L. Wen, H. Zheng, W. Li and X.-F. Peng, The relaxation modulus-based matrix splitting iteration method for solving linear complementarity problems of positive definite matrices, Appl. Math. Comput. 321, 349-357 (2018).

[27] S.-L. Wu and C.-X. Li, Two-sweep modulus-based matrix spliting iteration method for linear complementarity problems, J. Comput. Appl. Math. 302, 327-339 (2016).

[28] X.-P. Wu, X.-F. Peng and W. Li, A preconditioned general modulus-based matrix splitting iteration method for linear complementarity problems of H-matrices, Numer. Algorithms 79, 1131-1146 (2018).

[29] L.-L. Zhang and Z.-R. Ren, Improved convergence theorems of modulus-based matrix splitting iteration methods for linear complementarity problems, Appl. Math. Lett. 26, 638-642 (2013).

[30] L.-L. Zhang, Two-step modulus based matrix splitting iteration for linear complementarity problems, Numer. Algorithms 57, 83-99 (2011).

[31] H. Zheng, W. Li and S.-W. Vong, A relaxation modulus-based matrix splitting iteration method for solving linear complementarity problems, Numer. Algorithms 74, 137-152 (2017).

[32] N. Zheng and J.-F. Yin, Accelerated modulus-based matrix splitting iteration methods for linear complementarity problems, Numer. Algorithms 64, 245-262 (2013). 